\title{
Modelado de la interacción fluido estructura (FSI) para el diseño de una turbina eólica HAWT
}

\section{Modeling of the fluid structure interaction (FSI) for the design of a HAWT wind turbine}

\author{
Kevin Molina ${ }^{1}$, Daniel Ortega ${ }^{2}$, Manuel Martínez ${ }^{3}$, William Pinto-Hernández ${ }^{4}$, \\ Octavio Andrés González-Estrada ${ }^{5}$
}

\footnotetext{
${ }^{1}$ Grupo de Investigaciones Ciencias Básicas Aplicadas (Gicba), Unidades Tecnológicas de Santander, Bucaramanga, Colombia. Email: kevinandrey212@gmail.com

${ }^{2}$ Grupo de Investigaciones Ciencias Básicas Aplicadas (Gicba), Unidades Tecnológicas de Santander, Bucaramanga, Colombia. Email: daoa-1995@hotmail.com

${ }^{3}$ Grupo de Investigación en Energía y Medio Ambiente (Giema), Escuela de Ingeniería Mecánica, Universidad Industrial de Santander, Bucaramanga, Colombia. Email: mjmartin@uis.edu.co

${ }^{4}$ Grupo de Investigación en Energía y Medio Ambiente (Giema), Escuela de Ingeniería Mecánica, Universidad Industrial de Santander, Bucaramanga, Colombia. Email: wpintoh@uis.edu.co

${ }^{5}$ Grupo de Investigación en Corrosión (GIC), Escuela de Ingeniería Mecánica, Universidad Industrial de Santander, Bucaramanga, Colombia. Orcid: 0000-0002-2778-3389. Email: agonzale@uis.edu.co
}

Recibido: febrero 14, 2018. Aceptado: mayo 2, 2018. Versión final: mayo 28, 2018.

\begin{abstract}
Resumen
En este trabajo se estudia el comportamiento mecánico del álabe de una turbina eólica, analizando el impacto del diseño del álabe en la cantidad de energía generada, y su respuesta estructural en función de las fuerzas inducidas por el flujo de aire. Para estudiar la relación entre las fuerzas del flujo, los desplazamientos y esfuerzos provocados en la estructura, utilizamos un modelo de interacción fluido estructura (FSI). La estructura del álabe se diseña por medio de la teoría del momento y el elemento de pala (BEM), tomando como puntos de partida constantes referentes al viento (velocidad, densidad, Reynolds) y la selección de un perfil recomendado por la NACA, RISO, DU o NREL. Posteriormente, se efectúa el mismo procedimiento para un perfil diseñado mediante teorías de desempeño. Teniendo los resultados de estos dos tipos de álabes, se realiza una comparación de los esfuerzos y desplazamientos debidos a las variaciones entre el perfil diseñado y el perfil seleccionado, estudiando cómo esta variación afecta la energía generada de la turbina y su integridad estructural.
\end{abstract}

Palabras clave: álabe; BEM; diseño de perfiles; FSI; turbina eólica; HAWT.

\footnotetext{
Abstract

In this work, the mechanical behavior of the blade of a wind turbine is studied, analyzing the impact of the design of the blade on the amount of energy generated and its structural response as a function of the stresses induced by the air flow. In order to study the relationship between flow forces, deformations and stresses induced in the structure, a Fluid Structure Interaction (FSI) model was used. The blade structure is designed using blade element momentum theory (BEM), taking as a starting point constant properties related to the wind (speed, density, Reynolds), and the selection 
of a profile recommended by NACA, RISO, DU or NREL. Then, the same procedure is used for a profile designed using performance theories. Having these results for both types of blades, a comparison of the stresses and deformations due to the variations between the designed profile and the selected profile was made, analyzing the effects on the generated energy and the structural integrity of the turbine.

Keywords: blade; BEM; FSI; HAWT; profile design; wind turbine.

\section{Introducción}

El estudio de las energías renovables es actualmente un área prioritaria de investigación en ingeniería [1], [2]. En el área de aerogeneración, el diseño y modelado de álabes es uno proceso fundamental, no solo por el hecho de que se invierte mucho tiempo y dinero en prototipos y sus posteriores estudios, sino porque de este diseño depende la cantidad de energía que se conseguirá del aire [1], [3]. Esto hace que la variación de los parámetros de un álabe tenga gran influencia sobre la cantidad de energía generada, ya sea por la alteración de la geometría de sus perfiles, o como tal por su estructura [4], [5].

Zhu y Shen [6] exponen el desarrollo de diferentes pasos en el diseño por optimización de la estructura de un álabe, con ayuda del método de momento y el elemento de pala, BEM, y algunas correcciones propuestas por Prandtl para este método. Asimismo, el Laboratorio Nacional de Energía Renovable (NREL) [7] muestra el uso de las teorías de diseño de álabes utilizando BEM, y aplica nuevos métodos como el generalized dynamic wake (GDW). Dykes et al. [8] también presentan una nueva propuesta de optimización, no solo de la estructura del álabe, sino que abarca el desarrollo de toda una planta aerogeneradora, la cual optimiza desde la estructura de la turbina y todos sus componentes, hasta el costo de esta y su energía generada.

Recientemente, Phelps y Singleton [9] exponen el diseño y optimización de una turbina eólica de eje horizontal, HAWT, que con ayuda de un modelo de interacción fluido estructura, FSI, desarrolla el diseño de un larguero de soporte en el álabe, lo que disminuye la cantidad de material y, por ende, el costo de este, de forma que se obtienen los espesores de la superficie del álabe, de su larguero y su costo. Con las mismas herramientas, Stout et al. [10] comparan la eficiencia de un rotor de eje vertical, VAWT, optimizado con respecto al original.

Este trabajo analiza el impacto de la variación geométrica de los perfiles de una turbina eólica HAWT, por medio de la interacción fluido estructura, utilizando el método BEM para el desarrollo de los álabes y un proceso de optimización de forma por sensibilidad para el desarrollo de un álabe con perfiles optimizados. También se comparan los resultados con una turbina Nordex N120.

\section{2. Álabes}

Los álabes son la esencia de un aerogenerador, ya que de estos depende la cantidad de energía que se obtendrá del viento [11]. Por lo general están divididos en 4 secciones con una longitud en función de la envergadura del álabe [12] (raíz de pala, $12 \%$; interna, 25 \%; central, 50\%, y externa, 25\%), como se muestra en la figura 1.

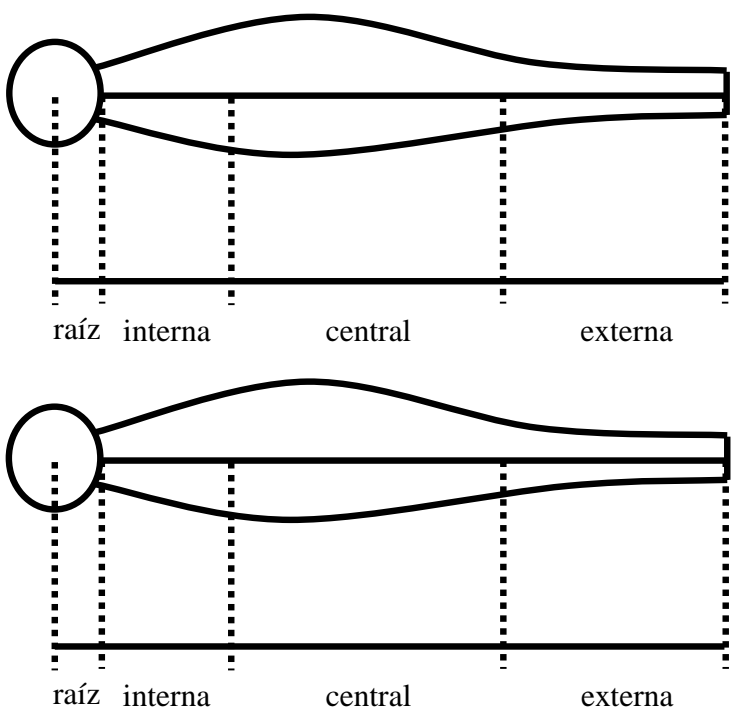

Figura 1. Secciones de un álabe. Fuente: elaboración propia.

\subsection{Perfiles aerodinámicos}

El corte transversal de cada sección está dado por lo que se conoce como perfil aerodinámico, del que dependen las fuerzas aerodinámicas de sustentación $L$ y arrastre $D$, así como sus respectivos coeficientes de sustentación y arrastre $C_{l}$ y $C_{d}$, respectivamente. Entre secciones se produce una variación de estos perfiles buscando hacer más eficiente el trabajo del álabe, esto se logra escogiendo los perfiles según la función que cumplen en cada una de las secciones.

La raíz de la pala cumple una función estructural, por lo que para esta se necesita un perfil con un área considerable que soporte altos esfuerzos. Por otro lado, la sección interna, aunque debe soportar esfuerzos, cumple un papel de generador de energía a muy bajas velocidades, y se hace necesario un perfil con un $C_{l}$ alto 
para estas condiciones. La parte más larga del álabe es la parte central y se encarga de sacar el mayor provecho a la energía del viento. Una de las características de sus perfiles es que tienen una relación de $C_{l} / C_{d}$ alta, esto con el fin de generar mayor energía, además de que estos perfiles tienen un ángulo elevado de entrada en pérdida, ya que, al ser la parte más larga del álabe, deben poseer una región amplia en la que puedan variar los ángulos de incidencia de sus perfiles. Por último, la parte externa que, además de generar energía, debe disminuir las pérdidas producidas por diferencias de presiones al final de la punta. Sus perfiles poseen elevados coeficientes de sustentación, pero bajos coeficientes de arrastre para disminuir las perdidas mencionadas.

Bertagnolio [13] proporciona algunas recomendaciones de perfiles NACA serie 6 utilizados generalmente en aerogeneradores, además de gráficas de coeficientes de sustentación y arrastre en función de su ángulo de incidencia. Esta serie contiene 6 números para su caracterización. Como ejemplo, tenemos el NACA 63215 , donde el primer número, " 6 ", hace referencia a la serie del perfil; el segundo número, "3", dice la localización de la presión mínima en función de la cuerda; el tercer número, "2", es el coeficiente de sustentación de diseño, los últimos dos dígitos, "15", representan el máximo espesor de la cuerda. Los perfiles recomendados para aerogeneradores de eje horizontal son los siguientes:

- NACA 63-215, NACA 63-218, NACA 63-221.

- NACA 63-415, NACA 63-418, NACA 63-421.

- NACA 64-415, NACA 64-421.

- NACA 65-415, NACA 65-421.

Se realizaron las polares de cada uno con ayuda del programa Javafoil [14], de donde se consiguieron los valores de $C_{l}$ y $C_{d}$ para diferentes ángulos de ataque $\alpha$ (entre $0^{\circ}$ y $30^{\circ}$ ) y Reynolds (entre 50000 y 100000). Con estos valores se escogieron manualmente los perfiles teniendo en cuenta las diferentes características para cada sección. Para la sección interna se obtuvo el perfil NACA 64-421 que tiene un alto $C_{l}$ a bajos Reynolds y $\alpha\left(5^{\circ} \mathrm{a}\right.$ $10^{\circ}$ ), teniendo además la mayor área con respecto a los demás perfiles. Para la sección media se eligió el NACA 63-421 con el $C_{l}$ más alto a diferentes Reynolds y $\alpha$. Por último, en la sección externa se decidió por el NACA 63221 por tener un bajo $C_{d}$ a altos Reynolds y $\alpha\left(15^{\circ}\right.$ a $\left.30^{\circ}\right)$.

Para la optimización de perfiles se tomó como punto de partida los perfiles escogidos para cada sección del álabe. En cada uno se optimizó un parámetro diferente, dependiendo de su función ya descrita, mejorando en el perfil interior su $C_{l}$ a bajas velocidades $(7 \mathrm{~m} / \mathrm{s})$, en el medio una mejor relación entre el $C_{l}$ y $C_{d}$ y, por último, en el exterior un menor $C_{d}$ sin disminuir su $\mathrm{C}_{\mathrm{l}}$.

En la optimización de estos perfiles se usó un método de sensibilidad con respecto a la geometría mediante el módulo Adjoint Solver del software ANSYS [15]. En este caso la geometría es el perfil y la sensibilidad está dada por la relación del perfil con las fuerzas aerodinámicas. Los cambios en el perfil son producidos en los puntos de mayor sensibilidad con el fin de disminuir o aumentar estas fuerzas y que su cambio con respecto al cambio geométrico sea mínimo, de modo que se obtenga un perfil optimizado.

\subsection{Método BEM}

El método BEM es muy utilizado en la actualidad para el desarrollo de la geometría de hélices. Este se fundamenta a partir de la relación de las teorías de momento y del elemento de pala, haciendo uso de parámetros referentes a los perfiles aerodinámicos, la geometría de la turbina (número de aspas, envergadura de los álabes) y el viento (velocidad, viscosidad cinemática).

\subsubsection{Teoría de momento}

Una manera de explicar la teoría de momento es mediante el modelo de Rankine-Froude [7], [15]. Esta toma la hélice como un disco infinitamente delgado y divide el campo de flujo en 4 secciones, mostradas en la figura 2. En la primera sección, el viento entra con velocidad $V_{1}$, presión $P_{1}$ y en estado laminar. A medida que avanza hacia la sección 2 este pierde velocidad hasta alcanzar $V_{2}$. En este punto atraviesa la hélice hasta la sección 3 , y no se produce un cambio significativo en la velocidad, por lo que $V_{2}=V_{3}=V$, pero sí se produce una variación de presión debido a la energía obtenida por la hélice $P_{2} \neq P_{3}$. El viento continúa avanzando hasta salir por la sección 4 con una velocidad $V_{4}$; aquí las presiones se igualan con la presión de entrada $P_{1}=P_{4}$.

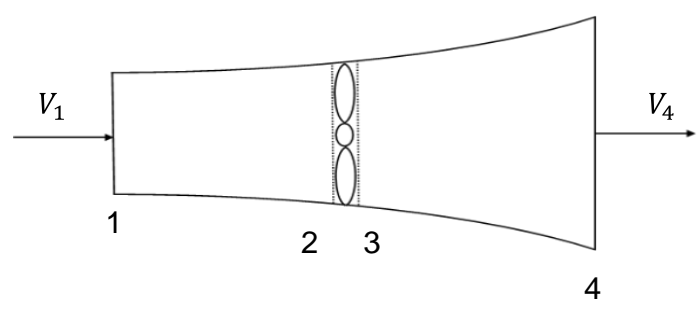

Figura 2. Modelo de Rankine-Froude. Fuente: elaboración propia.

Se utiliza el teorema de Bernoulli para analizar el modelo, esto no se puede realizar a lo largo del campo de flujo debido al salto de presión producido por la hélice, 
así que se realiza entre las secciones $1-2$ y 3-4, de lo que se obtienen las siguientes ecuaciones, donde $\rho$ es la densidad del aire:

$$
\begin{aligned}
& \frac{1}{2} \rho\left(V_{1}{ }^{2}-V_{2}{ }^{2}\right)=\left(P_{2}-P_{1}\right), \\
& \frac{1}{2} \rho\left(V_{3}{ }^{2}-V_{4}{ }^{2}\right)=\left(P_{4}-P_{3}\right) .
\end{aligned}
$$

Con las ecuaciones (1) y (2), y teniendo en cuenta las condiciones $V_{2}=V_{3}$ y $P_{1}=P_{4}$, se obtiene:

$$
\Delta P=P_{2}-P_{3}=\frac{1}{2} * \rho *\left(V_{1}^{2}-V_{4}^{2}\right) \text {. }
$$

Teniendo las diferencias de presión en la hélice se logra hallar la fuerza de empuje ejercida sobre esta:

$$
d F_{x}=\Delta P d A=\frac{1}{2} \rho\left(V_{1}^{2}-V_{4}^{2}\right) d A .
$$

Además, por la ecuación de cantidad de movimiento axial y de continuidad de caudal:

$$
\begin{gathered}
F_{x}=\int_{A}^{0} V \rho(V d A), \\
F_{x}=V_{4} \rho V_{4} A_{4}-V_{1} \rho V_{1} A_{1}, \\
\text { Caudal }=\rho V_{4} A_{4}=\rho V_{1} A_{1}=\rho V_{2} A,
\end{gathered}
$$

Se obtiene la ecuación:

$$
d F_{x}=\rho V_{2}\left(V_{1}-V_{4}\right) d A .
$$

Con las ecuaciones (4) y (5) se obtiene:

$$
V_{2}=\frac{V_{1}+V_{4}}{2}
$$

Y al incluir un factor de inducción axial $a$ :

$$
\begin{gathered}
a=\frac{V_{1}-V_{2}}{V_{1}}, \\
V_{2}=V_{1}(1-a),
\end{gathered}
$$

Se obtiene la ecuación (8), que elimina la variable $V_{4}$ de la ecuación (4):

$$
\begin{gathered}
V_{4}=V_{1}(1-2 a) \\
d F_{x}=\pi \rho V_{1}^{2}[4 a(1-a)] r d r .
\end{gathered}
$$

Ahora se analiza la cantidad de momento angular para la hélice, con el fin de hallar el torque generado sobre esta.

$$
T=\frac{d L}{d t} ; \quad \frac{d L}{d t}=\frac{d I}{d t} \omega ; \quad \frac{d I}{d t}=\frac{d m}{d t} r^{2} .
$$

Siendo $L$ el momento de inercia; $I$, el momento angular, y $\omega$, la velocidad angular inducida por la hélice sobre el viento. Así que:

$$
\begin{array}{r}
T=\frac{d m}{d t} r^{2} \omega, \quad \frac{d m}{d t}=\dot{m}=\rho A V_{2}, \\
d T=\rho V_{2} r^{2} \omega d A=2 \pi \rho V_{2} \omega r^{3} d r .
\end{array}
$$

Al igual que en las fuerzas de empuje, se introduce un factor de inducción radial $\left(a^{\prime}\right)$ a la ecuación (10) para eliminar la variable $\omega$ :

$$
a^{\prime}=\frac{\omega}{2 \Omega}
$$

Donde $\Omega$ es la velocidad angular de la hélice. Recordando la ecuación (7) para eliminar $V_{2}$ :

$$
d T=4 \pi \rho \Omega V_{1} a^{\prime}(1-a) r^{3} d r
$$

Teniendo así las ecuaciones que gobiernan las fuerzas de empuje (9) y los torques (12), por medio de la teoría de momento.

Antes de seguir con la teoría de elemento de pala se debe agregar a estas ecuaciones un coeficiente de corrección de pérdidas $Q$, debido que las fuerzas axiales y de torque son afectadas por los vórtices creados por las diferencias de presión en las puntas. Este coeficiente es estudiado por diferentes autores $y$, en este caso, se escoge el planteamiento de Prandtl:

$$
Q=\frac{2}{\pi} \cos ^{-1}\left(e^{-\frac{\frac{B}{2}\left(1-\frac{r}{R}\right)}{R} \operatorname{sen}(\phi)}\right)
$$

Donde $B$ es el número de álabes, $R$ es la envergadura del álabe y $\phi$ es el ángulo de incidencia. El coeficiente se multiplica por las ecuaciones (9) y (12):

$$
\begin{aligned}
& d F_{x}=\pi \rho Q V_{1}^{2}[4 a(1-a)] r d r \\
& d T=4 \pi Q \rho \Omega V a^{\prime}(1-a) r^{3} d r .
\end{aligned}
$$

\subsubsection{Teoría del elemento de pala}

La teoría del elemento de pala divide la hélice en elementos diferenciales para hallar las fuerzas ejercidas sobre cada uno, sin tener en cuenta los efectos de las secciones contiguas. Por lo general, el álabe es dividido en 10 a 20 elementos, cada uno de estos asociado a una velocidad de flujo $\Omega r$, cuerda $c$ y ángulo de incidencia $\phi$ diferente, con el fin de aprovechar mejor la energía del viento. 
El análisis comienza definiendo los ángulos de incidencia de cada sección. Para esto es necesario recordar que sobre la velocidad del aire que incide sobre el perfil $V_{w}$ se genera una velocidad angular $\omega$, producida por $\Omega$ y en dirección contraria a esta, como se muestra en la figura 3. Así que al analizar las velocidades tangenciales del viento que golpean el perfil se produce una velocidad relativa $V_{t r}$, mostrada en la figura 4. Junto con la velocidad axial del viento definen $\phi$ en la ecuación:

$$
\tan (\phi)=\frac{V_{1}(1-a)}{\Omega r\left(1+a^{\prime}\right)}
$$

Un factor importante en el diseño de álabes es la relación de velocidades locales de punta $\lambda_{r}$, la cual se hallará de manera gráfica más adelante, con ayuda del coeficiente de potencia y el tipo de rotor que se va a utilizar, el cual resulta de la ecuación anterior:

$$
\lambda_{r}=\frac{\Omega \mathrm{r}}{V_{1}}
$$

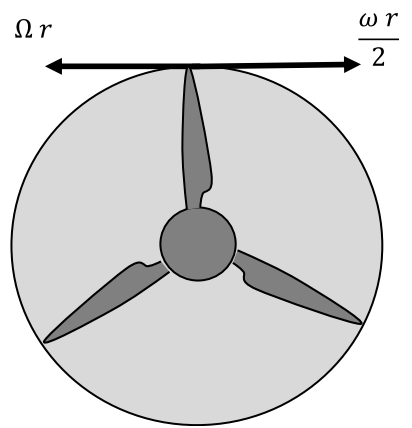

Figura 3. Velocidades tangenciales de la hélice y el viento. Fuente: elaboración propia.

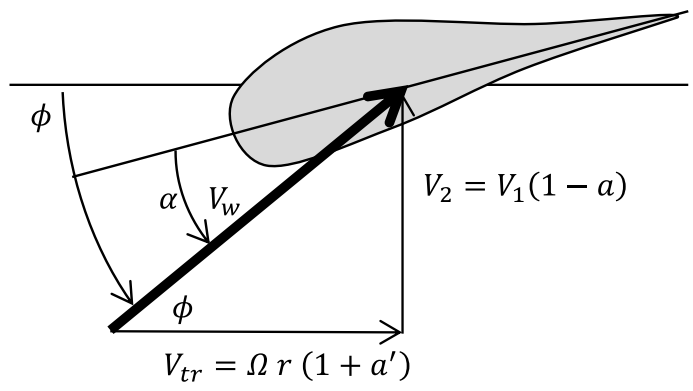

Figura 4. Descomposición de las velocidades del viento sobre el perfil. Fuente: elaboración propia.

Ahora se procede a definir las fuerzas que actúan sobre el perfil, como se puede ver en la figura 5. Las fuerzas están asociadas a la dirección con la que el viento incide sobre el perfil (la dirección de la fuerza de sustentación $L$ es perpendicular y de arrastre $D$ horizontal). Descomponiéndolas tangencial y axialmente se tiene:

$$
\begin{aligned}
& d F_{\theta}=d L \operatorname{sen}(\phi)-d D \cos (\phi) \\
& d F_{x}=d L \cos (\phi)+d D \operatorname{sen}(\phi)
\end{aligned}
$$

Las fuerzas $L$ y $D$ se definen por sus coeficientes $C_{l}$ y $C_{d}$ :

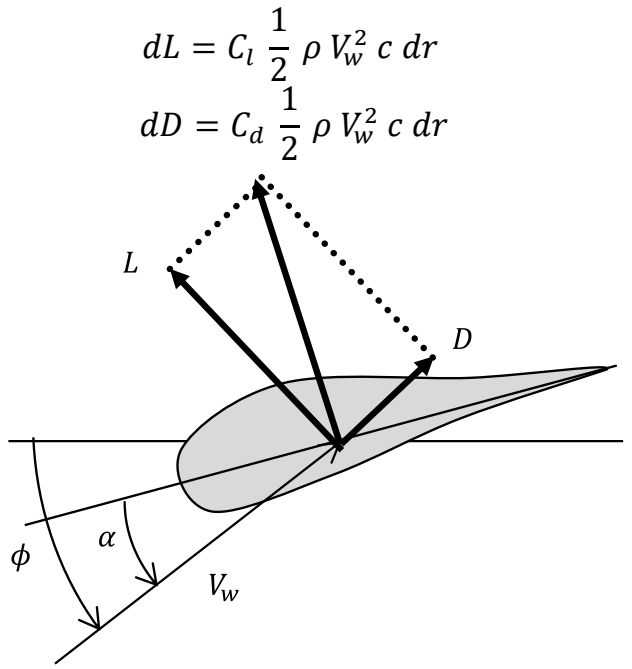

Figura 5. Fuerzas sobre el perfil. Fuente: elaboración propia.

Remplazando $d L$ y $d D$, y agregando el número de álabes $B$ en las ecuaciones (17) y (18) se obtiene:

$$
\begin{gathered}
d F_{\theta}=B \frac{1}{2} \rho V_{w}^{2} c\left(C_{l} \operatorname{sen}(\phi)\right. \\
\left.-C_{d} \cos (\phi)\right) d r \\
d F_{x}=B \frac{1}{2} \rho V_{w}^{2} c\left(C_{l} \cos (\phi)+C_{d} \operatorname{sen}(\phi)\right) d r .
\end{gathered}
$$

Al multiplicar la fuerza tangencial por el radio se obtiene el torque generado $d T$ :

$$
d T=B \frac{1}{2} \rho V_{w}^{2} c\left(C_{l} \operatorname{sen}(\phi)-C_{d} \cos (\phi)\right) r d r .
$$

Remplazando $V_{w}^{2}$ en $d T y d F_{x}$ se tienen las ecuaciones que gobiernan las fuerzas de empuje y las fuerzas radiales, por medio de la teoría del elemento de pala:

$$
\begin{array}{r}
d F_{x}=\sigma^{\prime} \pi \rho \frac{V_{1}^{2}(1-a)^{2}}{\operatorname{sen}^{2}(\phi)}\left(C_{l} \cos (\phi)\right. \\
\left.+C_{d} \operatorname{sen}(\phi)\right) r d r, \\
d T=\sigma^{\prime} \pi \rho \frac{V_{1}^{2}(1-a)^{2}}{\operatorname{sen}^{2}(\phi)}\left(C_{l} \operatorname{sen}(\phi)\right. \\
\left.-C_{d} \cos (\phi)\right) r^{2} d r,
\end{array}
$$

$\sigma^{\prime}$ es la solidez local

$$
\sigma^{\prime}=\frac{B c}{2 \pi r}
$$




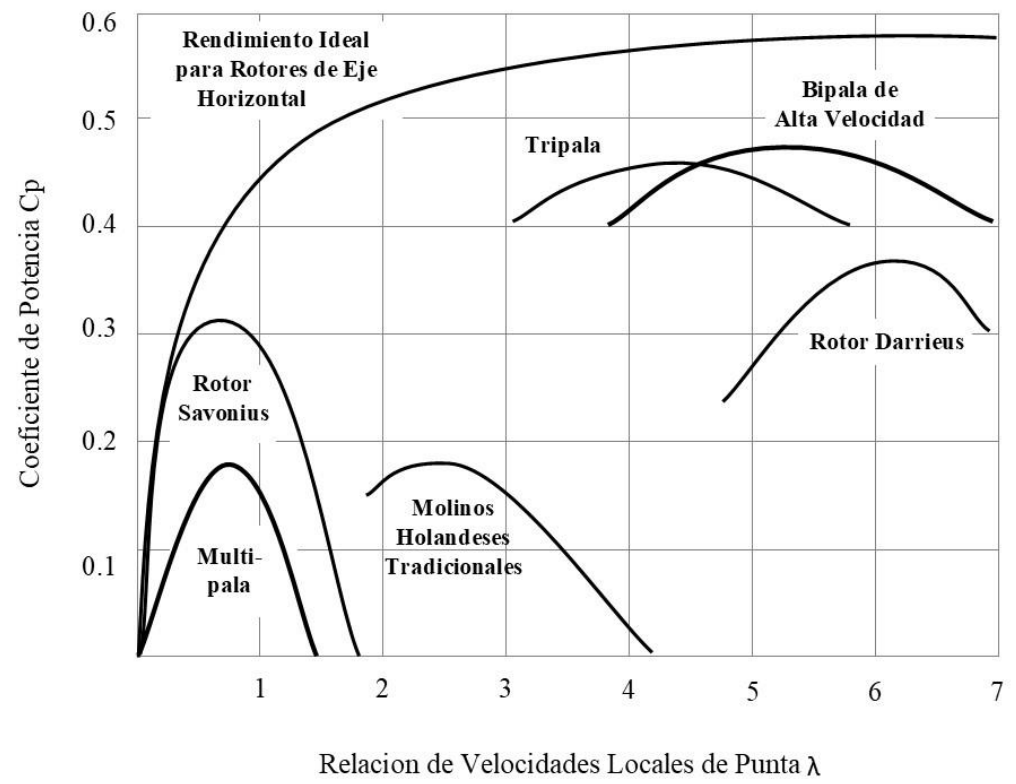

Figura 6. Rendimiento de hélices de molinos y turbinas de viento. Fuente: [18].

\subsubsection{Relación}

El método BEM relaciona dichas teorías dividiendo las ecuaciones de fuerza axial y las de torques, con el fin de despejar $a /(1-a)$ de las axiales y $a^{\wedge^{\prime}} /(1-a)$ de las tangenciales y tener las ecuaciones que definan los factores de inducción axial y tangenciales, con los cuales se hallarán la cuerda y ángulo de incidencia en cada sección:

$$
\begin{aligned}
\frac{a}{1-a} & =\frac{\sigma^{\prime}\left(C_{l} \cos (\phi)+C_{d} \operatorname{sen}(\phi)\right)}{4 Q \operatorname{sen}^{2}(\phi)} \\
\frac{a^{\prime}}{1-a} & =\frac{\sigma^{\prime}\left(C_{l} \operatorname{sen}(\phi)-C_{d} \cos (\phi)\right)}{4 Q \lambda_{r} \operatorname{sen}^{2}(\phi)}
\end{aligned}
$$

\subsection{Diseño de álabes}

Con ayuda de las ecuaciones planteadas por el método BEM, se comienza con el desarrollo del álabe. Este es un proceso iterativo en el que se supone un valor para los factores de inducción axial $a$ y tangencial $a^{\prime}$. Posteriormente, se desarrolla el método para el radio inicial de la sección interna del álabe y se halla $c y \phi$, con estos se recalcula $a y a^{\prime}$, se comparan estos con sus valores anteriores, si sus porcentajes de error son altos se realiza nuevamente la iteración con los nuevos valores.
Por el contrario, si estos son bajos se realiza el procedimiento para el siguiente radio.

Antes de comenzar a hallar $a y a^{\prime}$ se tendrán que definir algunos parámetros referentes a la geometría y el funcionamiento del generador (la envergadura de este $R$, el número de palas $B$ y su velocidad de rotación $\omega$ ). Como explica Ingram [16], la envergadura depende de la cantidad de potencia que se va a generar, y está dada por la siguiente ecuación:

$$
P=C_{P} \eta \frac{1}{2} \rho \pi R^{2} V_{1}^{3}
$$

En donde $C_{P}$ es el coeficiente de potencia esperado (0.424).[17], $\eta$ es la eficiencia mecánica y eléctrica (0.9) [18] y $V_{1}$ es la velocidad del viento. Así obtenemos la envergadura del álabe.

El número de álabes es escogido de acuerdo con la figura 6 , que está basada en prácticas experimentales. Teniendo $C_{p}$ y sabiendo que es una turbina HAWT, la gráfica nos indica que podemos utilizar dos o tres álabes (bipala o tripala). Aunque las turbinas de dos son más económicas, se escoge una de tres, debido a que esta presenta mayor estabilidad [19]. Asimismo, es posible hallar la relación de velocidades con la que podemos hallar $\Omega$ :

$$
\lambda=\frac{\Omega R}{V_{1}}
$$


Para hallar un valor inicial de $a y a^{\prime}$, Ingram [16] recomienda el uso de las siguientes ecuaciones:

$$
\begin{aligned}
\phi=\frac{2}{3} \tan ^{-1}\left(\frac{1}{\lambda_{r}}\right) ; \quad & a=\left(1+\frac{4 \operatorname{sen}^{2}(\phi)}{\sigma^{\prime} C_{l} \cos (\phi)}\right)^{-1} ; \\
a^{\prime} & =\frac{1-3 a}{4 a-1}
\end{aligned}
$$

Para el cálculo de la cuerda:

$$
C=\frac{8 \pi r \operatorname{sen}(\phi)}{3 B \lambda_{r}}
$$

Desarrollando estas ecuaciones y la serie de iteraciones se parametriza la geometría del álabe.

\section{Resultados}

\subsection{Rediseño de perfiles}

Se realiza el modelado del campo de flujo sobre los perfiles seleccionados, para esto se escoge una geometría C-mesh utilizada comúnmente en el análisis de perfiles aerodinámicos [20] y mostrada en la figura 7 para el perfil NACA 63-221.

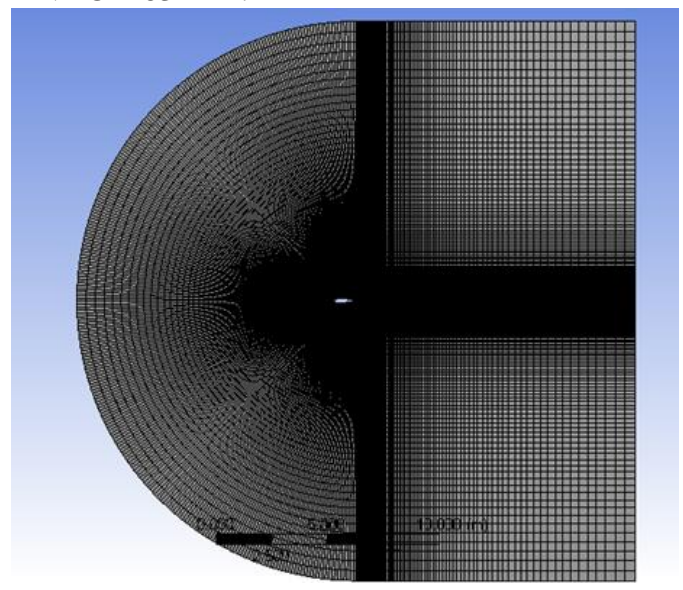

Figura 7. C-mesh del perfil NACA 63221. Fuente: elaboración propia.

Las condiciones de contorno utilizadas son las velocidades de entrada del aire. Estas tienen una magnitud y ángulo de ataque variante de un perfil a otro, debido a la velocidad angular de la pala y al ángulo de mayor aprovechamiento de energía, los cuales se observan en la tabla 1. Las velocidades de entrada actúan sobre los bordes inlet. Se tomó el borde airfoil como tipo Wall, y el borde outlet como Pressure-outlet. Estos se muestran la figura 8 .
Tabla 1. Velocidades y ángulos de ataque del viento sobre los perfiles de cada sección.

\begin{tabular}{lcc}
\hline Perfil & $\begin{array}{c}\text { Velocidad } \\
{[\mathbf{m} / \mathbf{s}]}\end{array}$ & $\begin{array}{c}\text { Ángulo } \\
{\left[{ }^{\circ}\right]}\end{array}$ \\
\hline NACA 64-421 & 10 & 5 \\
NACA 63-421 & 30 & 5 \\
NACA 63-221 & 60 & 5 \\
\hline
\end{tabular}

Fuente: elaboración propia.

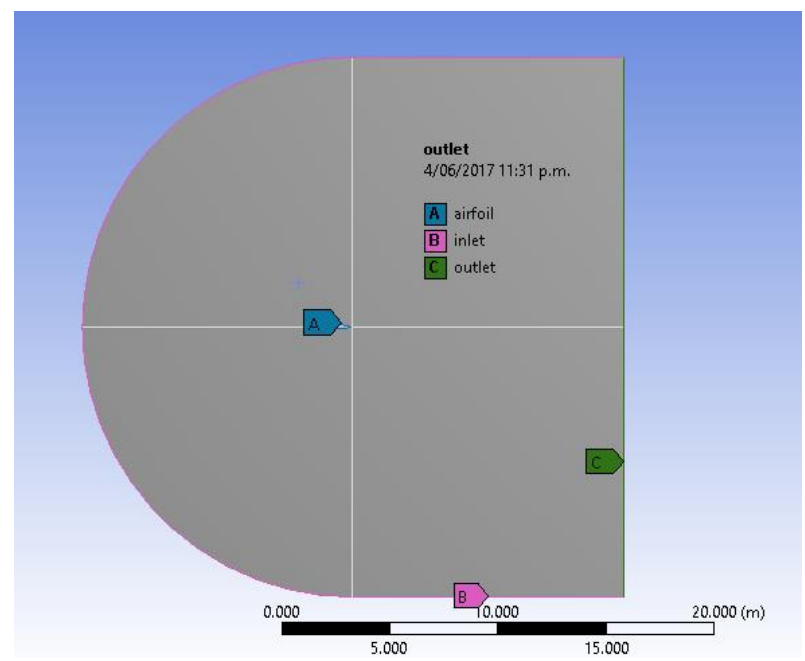

Figura 8. Bordes del campo de flujo. Fuente: elaboración propia.

Para el mallado se realiza un análisis de independencia de malla a este mismo perfil, para ver la convergencia de la solución, teniendo como criterio $C_{l}$ y $C_{d}$, y un error mínimo de $2 \%$, los resultados se observan en la figura 9 y la tabla 2; además se puede apreciar cómo estos coeficientes se acercan a su valor real [13].

Tabla 2. Convergencia de los coeficientes aerodinámicos para el perfil NACA 63-221.

\begin{tabular}{lccll}
\hline $\begin{array}{l}\text { Número } \\
\text { elementos }\end{array}$ & $\boldsymbol{C}_{\boldsymbol{l}}$ & $\boldsymbol{C}_{\boldsymbol{d}}$ & $\begin{array}{l}\text { Error } \\
\boldsymbol{C}_{\boldsymbol{l}}\end{array}$ & $\begin{array}{l}\text { Error } \\
\boldsymbol{C}_{\boldsymbol{d}}\end{array}$ \\
\hline 80 & 0.6627 & 0.10959 & - & - \\
90 & 0.7753 & 0.10105 & $17 \%$ & $8 \%$ \\
100 & 0.8497 & 0.09648 & $10 \%$ & $5 \%$ \\
110 & 0.9033 & 0.09391 & $6 \%$ & $3 \%$ \\
120 & 0.9467 & 0.09186 & $5 \%$ & $2 \%$ \\
130 & 0.9861 & 0.09007 & $4 \%$ & $2 \%$ \\
140 & 1.0096 & 0.08892 & $2 \%$ & $1 \%$ \\
\hline
\end{tabular}

Fuente: elaboración propia. 


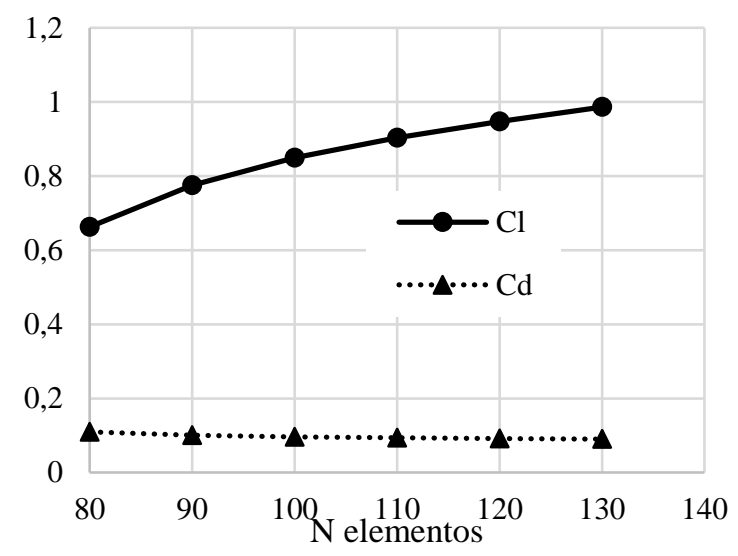

Figura 9. Convergencia de los coeficientes aerodinámicos para el perfil NACA 63-221. Fuente: elaboración propia.

Se usan ángulos de ataque de 5 grados, debido a que al usar ángulos mayores el $C_{d}$ del perfil aumenta, lo que produce una mayor fuerza de arrastre, que afecta el propósito de la turbina, además de que genera vibraciones indeseadas.

Teniendo la solución del campo de flujo, se realizó el proceso de optimización. En este se tomó $1 \%$ como el valor de cambio entre cada una de las iteraciones, y se realizó un cálculo total cada 5 iteraciones, para tener un control sobre la deformación del perfil y verificar el cambio del valor de $C_{l}$ y $C_{d}$. Los resultados se muestran en la tabla 3.

Tabla 3. Comparación de los coeficientes aerodinámicos con los perfiles optimizados.

\begin{tabular}{lllll}
\hline Perfiles & $\begin{array}{l}\boldsymbol{C}_{\boldsymbol{l}} \\
\text { inicial }\end{array}$ & $\begin{array}{l}\boldsymbol{C}_{\boldsymbol{l}} \\
\text { final }\end{array}$ & $\begin{array}{l}\boldsymbol{C}_{\boldsymbol{d}} \\
\text { inicial }\end{array}$ & $\begin{array}{l}\boldsymbol{C}_{\boldsymbol{d}} \\
\text { final }\end{array}$ \\
\hline NACA 63 & 1.018 & 1.06 & 0.892 & 0.088 \\
$\mathbf{2 2 1}$ & & & & \\
NACA 63 & 1.465 & 1.506 & 0.051 & 0.054 \\
$\begin{array}{l}\text { 221 } \\
\text { NACA 64 }\end{array}$ & 1.295 & 1.321 & 0.041 & 0.042 \\
$\mathbf{4 2 1}$ & & & & \\
\hline
\end{tabular}

Fuente: elaboración propia.

Se aprecia que los $C_{l}$ aumentaron para cada perfil según su condición de trabajo. Esta se tomó como un promedio entre secciones. Además, vemos que el $C_{d}$ para el NACA 63-221 disminuye como era requerido.

\subsection{Modelado del álabe}

Los parámetros iniciales para el desarrollo del álabe fueron tomados de las turbinas Nordex N120 [17] de
$1300 \mathrm{KW}$ con una velocidad de viento promedio de $8 \mathrm{~m} / \mathrm{s}$ [21]. Esta velocidad es la presente en el parque eólico Jepirachi [22], ubicado en la Guajira, Colombia.

Con estos valores de potencia y velocidad, se desarrolló el método BEM, con los perfiles seleccionados, teniendo como resultado la caracterización del álabe, dichas características se muestran en la tabla 4.

Para el centro de rotación de los perfiles se tomó una distancia de 0.30 veces de la cuerda a partir del borde de ataque, debido a que en dicho punto (línea de presión) inciden las fuerzas aerodinámicas [19]. También se implementó un larguero de soporte a lo largo del álabe a la misma distancia del borde de ataque. Los álabes resultantes para los perfiles seleccionados y optimizados se muestran en la figura 10.
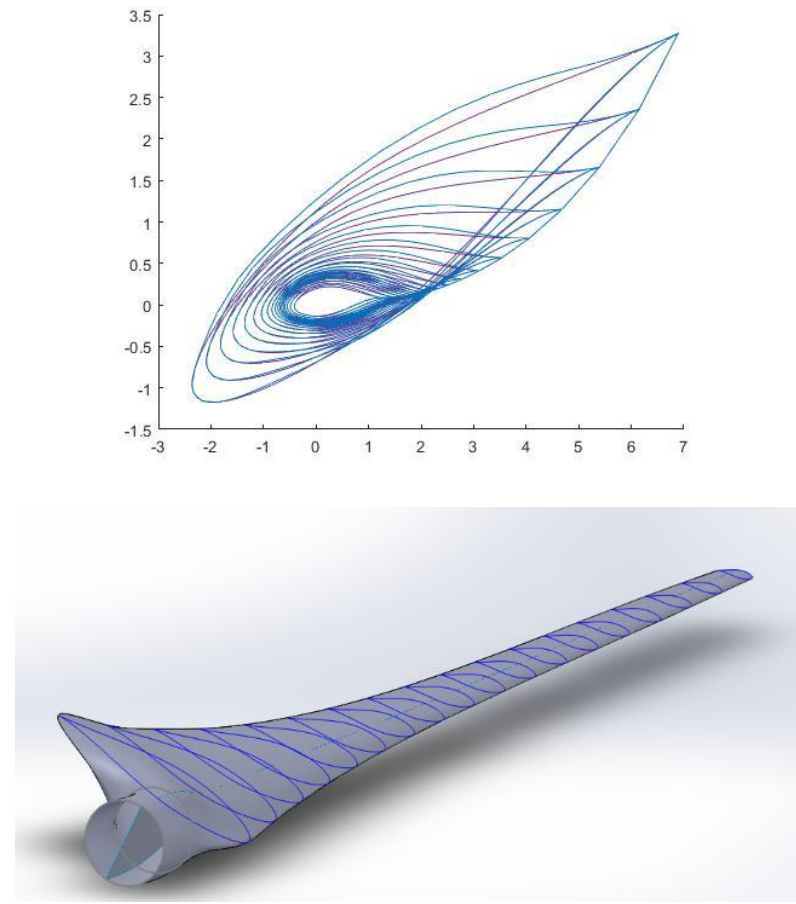

Figura 10. Álabes resultantes. (a) Perfiles seleccionados (morados) y optimizados (azules) y (b) estructura de los álabes. Fuente: elaboración propia.

\subsubsection{Velocidades y torque sobre los álabes}

Se realizó el campo de flujo sobre los álabes con un mallado de cono truncado, en el que solo se tomó un tercio de la turbina total y se analizó un solo álabe, debido a su simetría, con el fin de disminuir el gasto computacional. La malla se muestra en la figura 11. 
Tabla 4. Caracterización del álabe con perfiles seleccionados.

\begin{tabular}{llll}
\hline \multicolumn{1}{c}{$\mathbf{R}[\mathbf{m}]$} & $\begin{array}{c}\mathbf{\Phi} \\
{\left[{ }^{\circ}\right]}\end{array}$ & \multicolumn{1}{c}{$\mathbf{C}[\mathbf{m}]$} & $\begin{array}{r}\mathbf{V t a n} \\
{[\mathbf{m} / \mathbf{s}]}\end{array}$ \\
\hline 7.340 & 29.649 & 10.438 & 10.548 \\
10.399 & 24.475 & 8.877 & 13.143 \\
13.457 & 20.382 & 7.551 & 15.915 \\
16.516 & 17.300 & 6.440 & 18.811 \\
19.574 & 14.959 & 5.522 & 21.786 \\
22.633 & 12.819 & 4.777 & 24.812 \\
25.691 & 11.417 & 4.184 & 27.879 \\
28.750 & 10.281 & 3.721 & 30.973 \\
31.808 & 9.345 & 3.368 & 34.085 \\
34.867 & 8.561 & 3.103 & 37.213 \\
37.925 & 7.895 & 2.906 & 40.351 \\
40.984 & 7.321 & 2.755 & 43.498 \\
44.042 & 6.819 & 2.629 & 46.652 \\
47.101 & 6.368 & 2.508 & 49.811 \\
50.159 & 6.412 & 2.370 & 52.999 \\
53.217 & 5.958 & 2.194 & 56.161 \\
56.276 & 5.420 & 1.960 & 59.319 \\
59.334 & 4.423 & 1.646 & 62.442 \\
\hline
\end{tabular}

Fuente: elaboración propia.
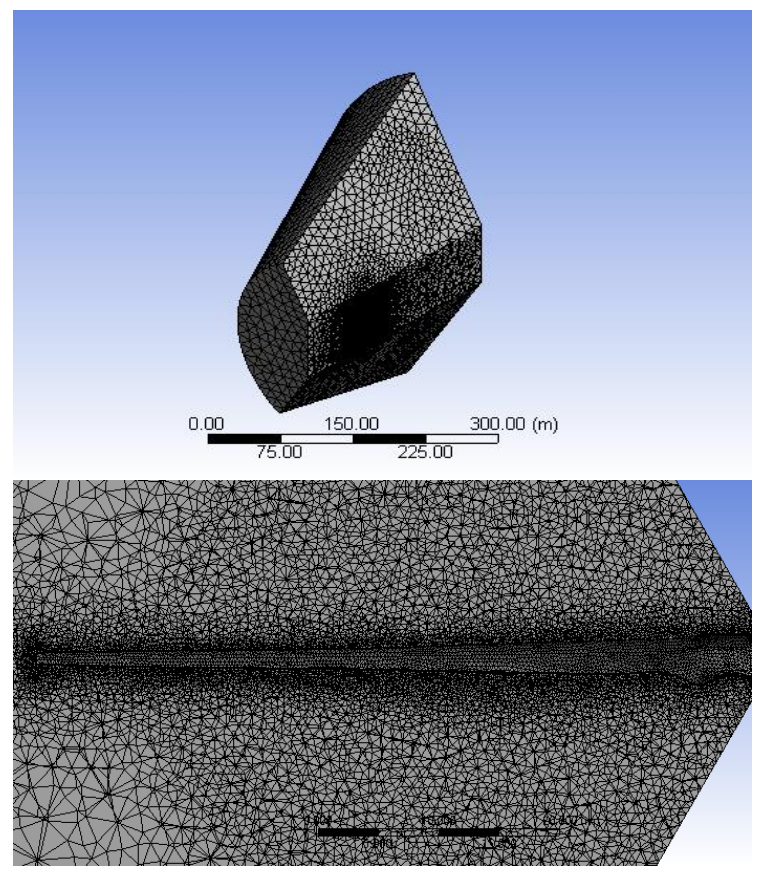

Figura 11. Mallado del campo de flujo. (a) Vista isométrica y (b) vista en detalle de la superficie en contacto con el álabe. Fuente: elaboración propia.

Para las condiciones de contorno se tomó la velocidad de entrada de $8 \mathrm{~m} / \mathrm{s}$, que actúan sobre las superficies inlet e inletTop, el contorno de salida outlet es tipo outlet- pressure, la superficie en contacto con el álabe blade es tipo Wall, y por ultimo las zonas de interfaz o de simetría periodl y period2 son de tipo interface. Estos bordes se muestran en la figura 13, que

La figura 13 muestra las velocidades tangenciales del álabe de los perfiles seleccionados, teniendo una velocidad en m/s máxima de 63 y mínima de 9.7 , que concuerdan con la velocidad obtenida por el método BEM de 62 y 10, respectivamente, con un error relativo del $1.6 \%$ y del $3 \%$, lo cual permite validar el mallado y las condiciones de contorno evaluadas anteriormente.

Al igual que con las velocidades tangenciales se obtuvieron y validaron los valores del torque generado para cada tipo de álabe, con el torque generado por una turbina de $1300 \mathrm{KW}$. Estos valores se pueden observar en la tabla 5. Como se observa, el torque generado por la turbina con los perfiles optimizados es mayor con respecto a la otra.

Tabla 5. Torques generados por las turbinas.

\begin{tabular}{lll}
\hline Turbina & $\begin{array}{l}\text { Torque } \\
{\left[\mathbf{N}^{*} \mathbf{m}\right]}\end{array}$ & Diferencia [\%] \\
\hline NORDEX & 1242829 & - \\
Original & 1260492 & $1.42 \%$ \\
Optimizada & 1313226 & $4.18 \%$ \\
\hline
\end{tabular}

Fuente: elaboración propia.

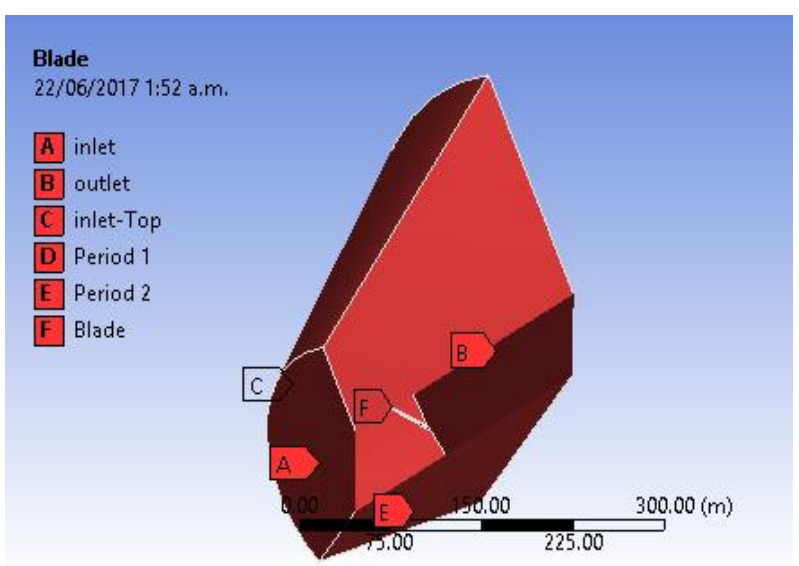

Figura 12. Bordes del campo de flujo. Fuente: elaboración propia.

La figura 13 muestra las velocidades tangenciales del álabe de los perfiles seleccionados, teniendo una velocidad en m/s máxima de 63 y mínima de 9.7, que concuerdan con la velocidad obtenida por el método BEM de 62 y 10, respectivamente, con un error relativo del $1.6 \%$ y del $3 \%$, lo cual permite validar el mallado y las condiciones de contorno evaluadas anteriormente. 


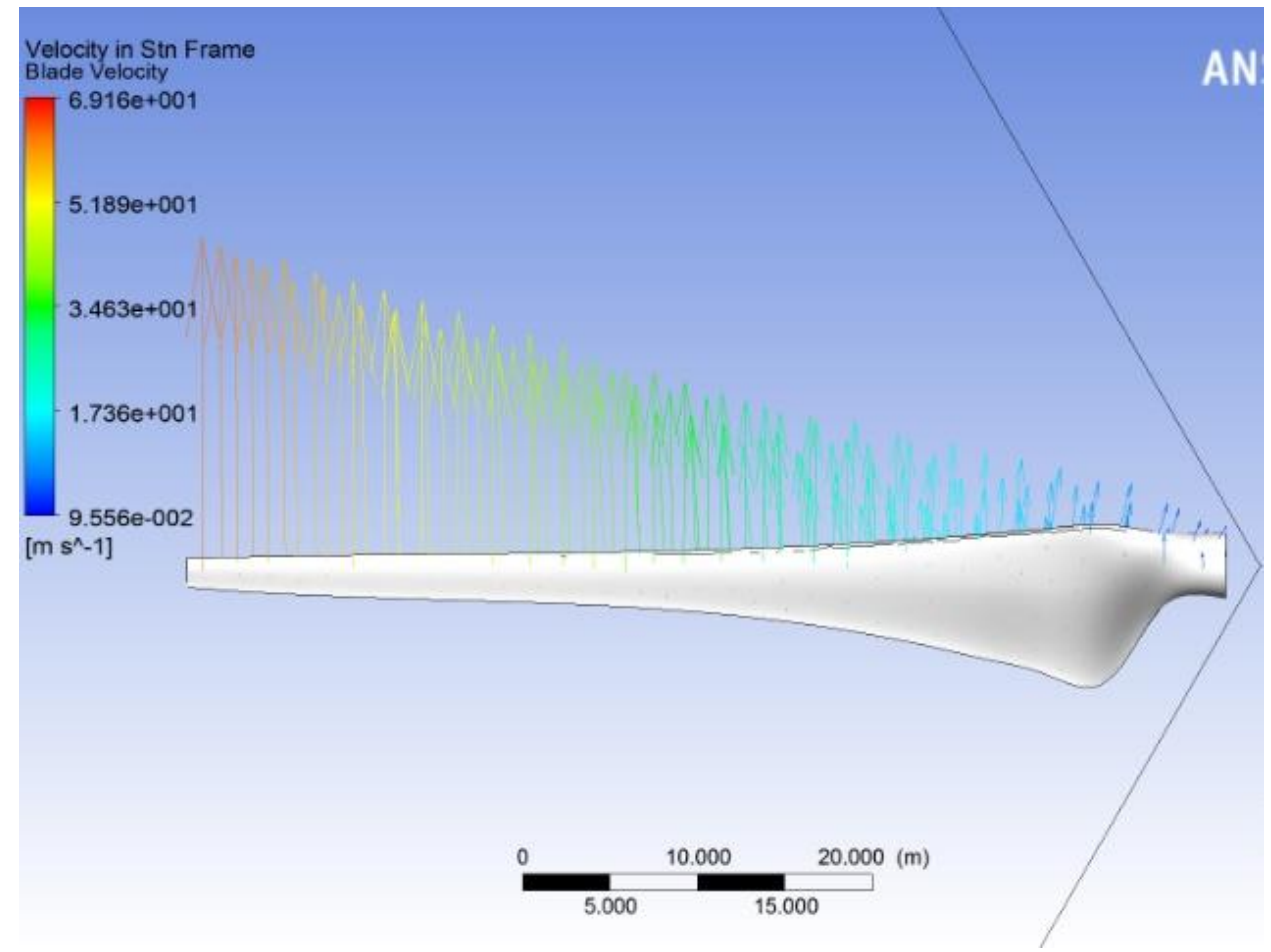

Figura 13. Velocidades a lo largo del álabe. Fuente: elaboración propia.

Al igual que con las velocidades tangenciales se obtuvieron y validaron los valores del torque generado para cada tipo de álabe, con el torque generado por una turbina de $1300 \mathrm{KW}$. Estos valores se pueden observar en la tabla 5. Como se observa, el torque generado por la turbina con los perfiles optimizados es mayor con respecto a la otra.

Tabla 5. Torques generados por las turbinas.

\begin{tabular}{lll}
\hline Turbina & $\begin{array}{l}\text { Torque } \\
{[\mathbf{N * m}]}\end{array}$ & Diferencia [\%] \\
\hline NORDEX & 1242829 & - \\
Original & 1260492 & $1.42 \%$ \\
Optimizada & 1313226 & $4.18 \%$ \\
\hline
\end{tabular}

Fuente: elaboración propia.

\subsubsection{Esfuerzos y deformaciones sobre álabes}

Para los álabes se utilizó un material ortótropo, utilizado comúnmente en la construcción de estas, y sus propiedades se muestran en la tabla 6. Los espesores seleccionados para los largueros y la superficie del álabe se encuentran en la tabla 7.
Tabla 6. Propiedades del material del álabe.

\begin{tabular}{ll}
\hline Propiedad & Valor \\
\hline Densidad $\left(\mathrm{kg} / \mathrm{m}^{\wedge} 3\right)$ & 1550 \\
Módulo de Young-X $(\mathrm{Pa})$ & $1.1375 \mathrm{E}+11$ \\
Módulo de Young-Y $(\mathrm{Pa})$ & 7583000000 \\
Módulo de Young-Z $(\mathrm{Pa})$ & 7583000000 \\
Relación de poisson-XY & 0.32 \\
Relación de poisson-YZ & 0.37 \\
Relación de poisson-XZ & 0.35 \\
Módulo de corte-XY $(\mathrm{Pa})$ & 5446000000 \\
Módulo de corte-YZ $(\mathrm{Pa})$ & 2964000000 \\
Módulo de corte-XZ $(\mathrm{Pa})$ & 2964000000 \\
\hline
\end{tabular}

Fuente: elaboración propia.

Los valores de las cargas de presión sobre los álabes son transferidos del modelo numérico del campo de flujo. Con estas se obtienen los esfuerzos de Von Mises y desplazamientos totales, como se observa en las figuras 14 y 15. Los esfuerzos son mayores sobre el álabe optimizado, debido a que las fuerzas y momentos que actúan sobre este son mayores espesores para la superficie del álabe y para el larguero. 

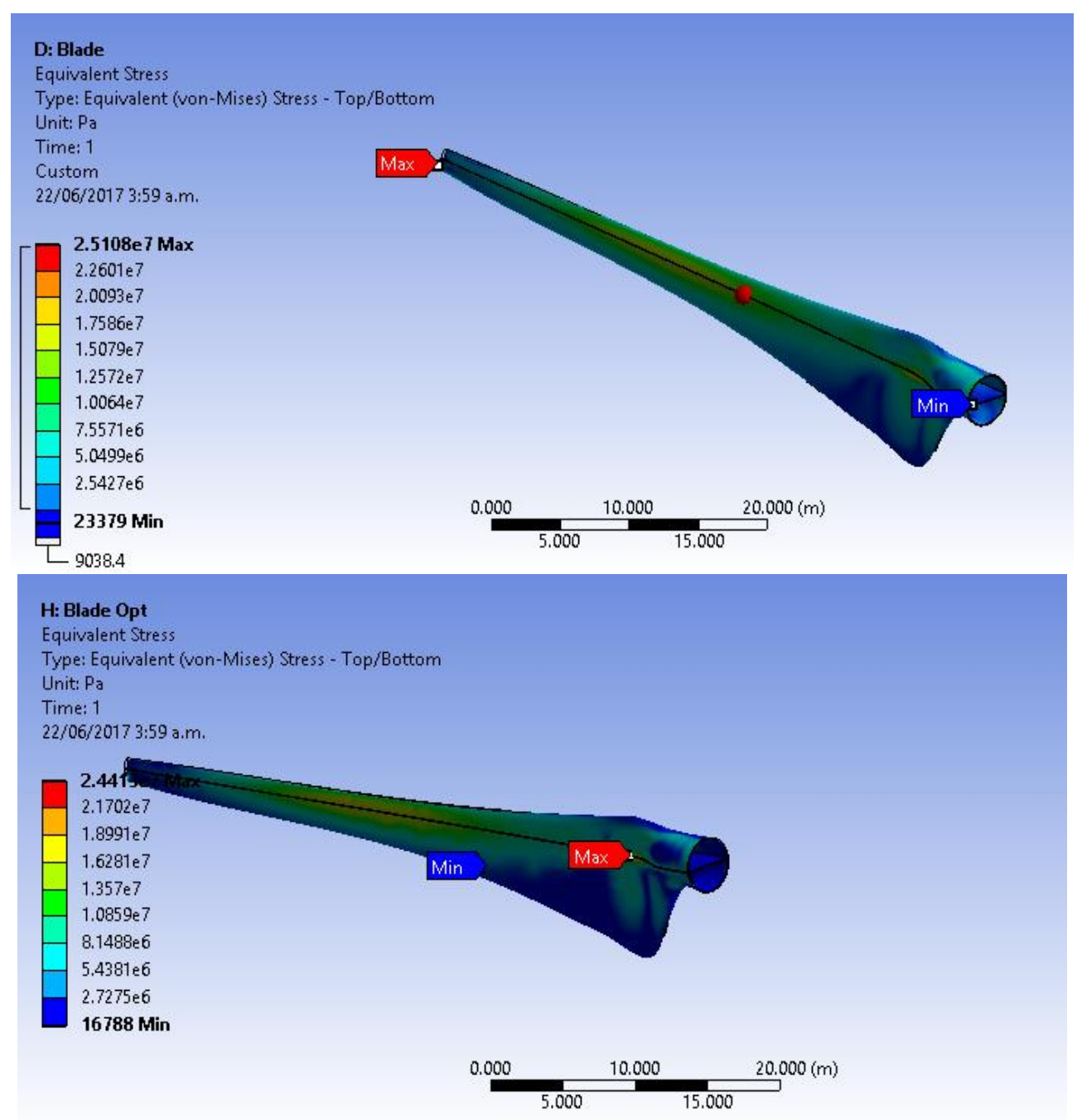

Figura 14. Esfuerzos sobre los álabes (a) original y (b) optimizado. Fuente: elaboración propia.

Tabla7. Espesores para la superficie del álabe y para el larguero.

\begin{tabular}{ll}
\hline Superficie del álabe & \\
\hline $\mathbf{X}(\mathbf{m})$ & Espesor $(\mathbf{m})$ \\
\hline-60 & 0.005 \\
-1.8 & 0.1 \\
\hline Larguero & \\
\hline $\mathbf{X}(\mathbf{m})$ & Espesor $(\mathbf{m})$ \\
\hline-1.8 & 0.1 \\
-60 & 0.03 \\
\hline
\end{tabular}

Fuente: elaboración propia.

\section{Conclusiones}

Se desarrolló un modelo de simulación numérica de la interacción fluido estructura del álabe y el campo de flujo, y se obtuvo el torque necesario para la validación del álabe optimizado y los desplazamientos y esfuerzos sobre este. Se pudo observar que los desplazamientos y esfuerzos son mayores en el álabe optimizado, debido que las fuerzas y momentos actuantes sobre este son mayores.

Se definió la geometría del álabe por medio del método BEM, validándolo con la geometría de una turbina N120 de $1300 \mathrm{KW}$, teniendo una diferencia mínima entre los principales parámetros, como son su envergadura y velocidad de rotación.

A partir de la selección de los perfiles para cada sección del álabe, y mediante un proceso de optimización de sensibilidad con respecto a la geometría, se obtuvieron álabes con un torque un $4.18 \%$ mayor al álabe inicial, con lo que se logró obtener mayor potencia a partir del cambio geométrico de los perfiles a lo largo del álabe. 

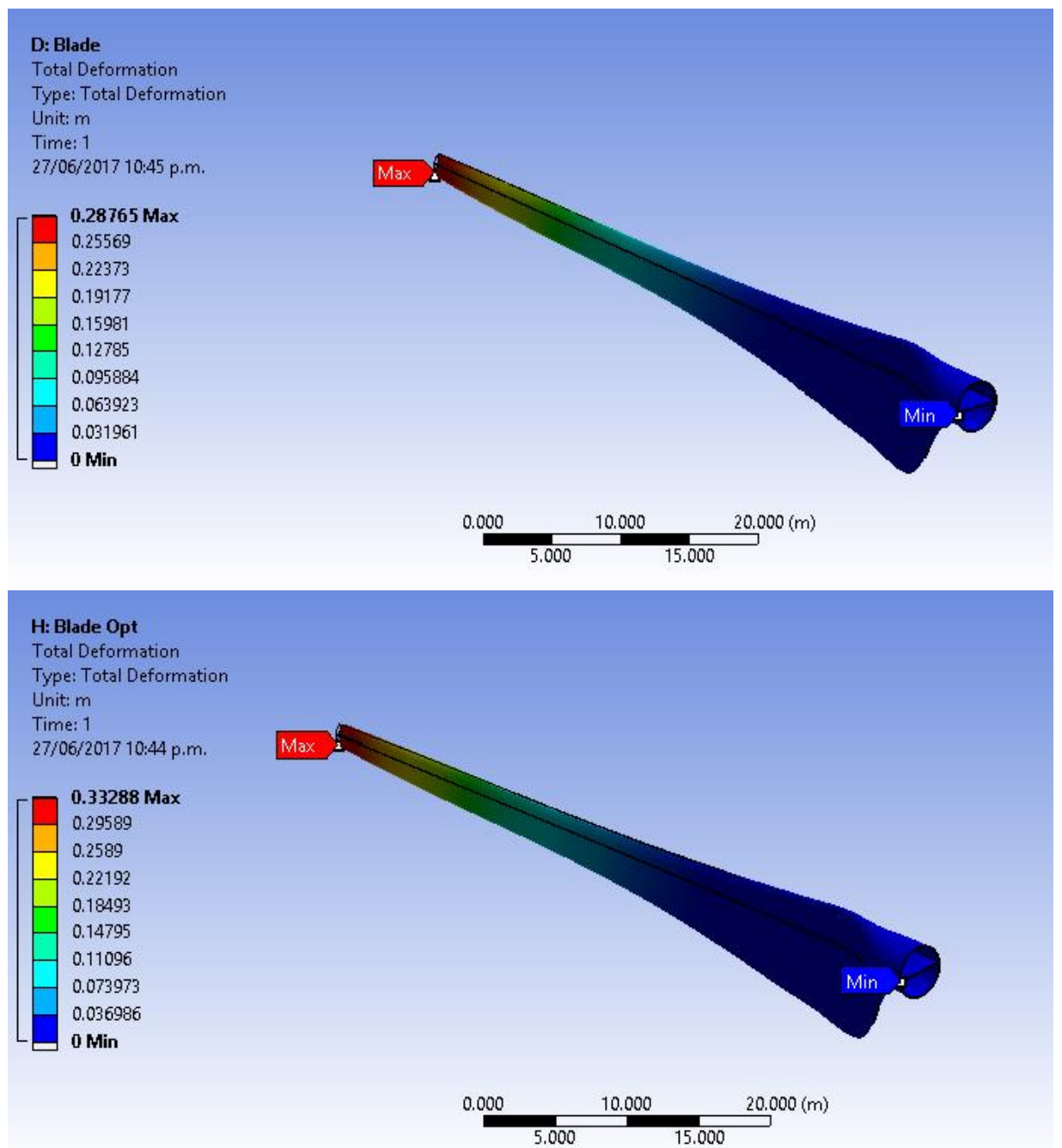

Figura 15. Desplazamientos de los álabes (a) original y (b) optimizado. Fuente: elaboración propia.

\section{Agradecimientos}

Este trabajo fue apoyado por el proyecto Capital Semilla 1742 de la Vicerrectoría de Investigación y Extensión, de la Universidad Industrial de Santander.

\section{Referencias}

[1] P. P. Vergara-Barrios, J. M. Rey-López, G. A. OsmaPinto, and G. Ordoñez-Plata, "Evaluación del potencial solar y eólico del campus centra de la Universidad Industrial de Santander y la ciudad de Bucaramanga, Colombia," Rev. UIS Ing., vol. 13, 2, pp. 49-57, 2014.

[2] Á. O. Díaz-Rey, J. E. González-Gil, O. A. GonzálezEstrada, Á. Díaz Rey, J. González Gil, and O. A. González-Estrada, "Análisis de un generador de HHO de celda seca para su aplicación en motores de combustión interna," Rev. UIS Ing., vol. 17, no. 1, pp. 143-154, 2018, doi:https://doi.org/10.18273/revuin.v17n1-2018013.

[3] A. Rodriguez, "Análisis cfd de un álabe del último paso de una turbina de vapor," Universidad Politecnica de Madrid, 2016.

[4] A. Ayestarán, C. Graciano, and O. A. GonzálezEstrada, "Resistencia de vigas esbeltas de acero inoxidable bajo cargas concentradas mediante elementos finitos," Rev. UIS Ing., vol. 16, no. 2, pp. 61-70, Sep. 2017, doi:10.18273/revuin.v16n2-2017006.

[5] E. E. Gaona, P. A. Mancera, C. L. Trujillo, and C. L. Trujillo Rodriguez, "Algoritmo de encaminamiento con reconfiguración de topología para red de sensores inalámbricos aplicada a una Microrred en modo "Isla ,"” Rev. UIS Ing., vol. 15, no. 2, pp. 93-104, Jan. 2016, 
doi:https://doi.org/10.18273/revuin.v15n2-2016008.

[6] W. J. Zhu and W. Z. Shen, "Integrated airfoil and blade design method for large wind turbines," in International Conference on aerodynamics of Offshore Wind Energy Systems and wakes (ICOWES2013), 2013, pp. $1-10$.

[7] P. J. Moriarty and A. C. Hansen, "AeroDyn Theory Manual," Golden, Colorado, 2005.

[8] K. Dykes et al., "Introducing WISDEM Integrated System Modeling for Wind Turbines and Plants," Golden, Colorado, 2014.

[9] C. Phelps and J. Singleton, "Wind Turbine Blade Design," Ithaca, NY, 2015.

[10] C. Stout et al., "Efficiency Improvement of Vertical Axis Wind Turbines with an Upstream Deflector," Energy Procedia, no. April, pp. 1-10, 2016.

[11] A. Lecuona Neumann, La Energía Eólica: Principios básicos y tecnología. Madrid: Universidad Carlos III de Madrid, 2002.

[12] R. van Rooij and N. Timmer, "Design of Airfoils for Wind Turbine Blades,” Delft, 2004.

[13] F. Bertagnolio, N. Sorensen, J. Johansen, and P. Fuglsang, "Wind turbine airfoil catalogue. Risø-R-1280 (EN)," Roskilde, Denmark, 2001.

[14] M. Hepperle, “JavFoil User's Guide.” pp. 1-45, 2014.

[15] D. Almazo, M. Toledo, M. Vega del Carmen, J. Abugaber, O. José Pineda, and A. Reyes, "Selección y diseño de hélices," in 15 Congreso Nacional de Ingeniería Electromecánica y de Sistemas, 2015, pp. 16.

[16] G. Ingram, "Wind Turbine Blade Analysis using the Blade Element Momentum Method. Version 1.1," Durham, 2011.

[17] Nordex, "N60 / 1300 kW. Long-term experience all over the world." Nordex Brochure.

[18] R. A. Bastianon, “Cálculo Y Diseño De la Hélice Óptima Para Turbinas Eólicas,” Buenos Aires, 2008.

[19] R. Gasch and J. Twele, Wind Power Plants: Fundamentals, Design, Construction and Operation, 2nd ed. Berlin, Heidelberg: Springer, 2012.
[20] A. Khare, A. Singh, and K. Nokam, "Best practices in grid generation for CFD application using HyperMesh," in ATC: HyperWorks Technology Conference 2009, 2009, pp. 1-10.

[21] IDEAM, “Atlas Interactivo - Vientos,” 2015.

[22] M. P. Rincón, "Parque eólico Jepirachi, Colombia | EJAtlas," 2014. 